\title{
In vitro gene silencing effect of chitosan/shRNA PDGF-D nanoparticles in breast cancer
}

\author{
Ceyda EKENTOK, Suna ÖZBAŞ TURAN, Jülide AKBUĞA
}

\begin{abstract}
Breast cancer is the most common cancer worldwide in women and it is highly malignant and fatal. PDGF-D plays role in regulation of many cellular processes such as angiogenesis. PDGF-D is overexpressed in many types of cancers and promote tumor growth and metastasis. Silencing of PDGF-D gene by using shRNA with an appropriate carrier system may decrease tumor growth and metastasis. In our study, we prepared chitosan nanoparticles loaded with five different shRNA plasmids targeting different exons of PDGF-D gene. Then, nanoparticles were characterized in vitro and transfection efficiency of these nanoparticles were investigated in breast cancer cell lines (MCF7, MDA-MB-231 and MDA-MB-435). The effects of single
\end{abstract}

and multiple shRNA sequences, molecular weight of chitosan $(150 \mathrm{kDa}$ and $400 \mathrm{kDa}$ ) and the amount of shRNA (100 and $500 \mu \mathrm{g}$ ) on the characterization and transfection efficiencies of nanoparticles have been studied. Size of nanoparticles changed between $200-400 \mathrm{~nm}$ and approximately $95-100 \%$ encapsulation efficiency were obtained. Release of shRNA changed with the molecular weight of chitosan. It was obtained that formulation containing shRNA plasmid targeting PDGF-D exon 6 (NP1) has the highest silencing efficiency in MDA-MB-231 cell line. It was also evaluated that chitosan can be a suitable gene delivery system for shRNA targeting PDGF-D.

Key words: Breast cancer ; chitosan ; shRNA ; gene silencing ; RNAi.
Ceyda Ekentok, Suna Özbaş Turan, Jülide Akbuğa

Department of Pharmaceutical Biotechnology, Faculty of Pharmacy, Marmara University, Haydarpaşa 34668 İstanbul, Turkey

\author{
Corresponding Author: \\ Ceyda Ekentok \\ e-mail: ceyda.ekentok@marmara.edu.tr
}

Submitted / Gönderilme: 05.05.2017 Revised / Düzeltme: -

Accepted / Kabul: 15.06 .2017

How to cite this article : Ekentok C, Özbaş Turan S, Akbuğa J. In vitro gene silencing effect of chitosan/shRNA PDGF-D nanoparticles in breast cancer. Marmara Pharm J 2017; 21 (4): 793-803

\section{Introduction}

Breast cancer is one of the most common, highly malignant and fatal disease. Angiogenesis, the process of new blood vessel formation, plays a critical role in solid tumor formation and growth. Thus, angiogenesis is an important target for cancer drug development.

Development and metastasis of tumors in the breast are dependent on angiogenesis. Various molecules such as vascular endothelial growth factor (VEGF), fibroblast growth factor (FGF), platelet-derived growth factor (PDGF) play roles in regulation of angiogenesis. Among them, VEGF and FGF are the most studied molecules [1].

In recent years, there are publications related to the effect of PDGF/PDGFR on tumor angiogenesis [2]. PDGFs belong to growth factor family that consist of 5 disulfide-linked dimmers (PDGF-A to D). PDGF molecules are mitogen for most cells and their expression increase in some malignant situations [3]. Platelet-derived growth factor signaling pathway is well-defined and recent years it has been discussed 
that PDGF signaling pathway is different in normal and cancer cells [4].

PDGF family consist of 4 different PDGF chains (-A, -B, -C, -D). PDGF-A and -B have been known since 1970, on the other hand PDGF-C has been found next years and finally PDGF-D has been found recently [5-7].

PDGFs regulate different cellular processes like cell proliferation, transformation, migration and viability during growth or pathogenesis $[8,9]$. Studies show that PDGF signal has an effect on growth, invasion and metastasis of cancer cells [10]. Increase of PDGF expression is generally related to tumor growth, angiogenesis and metastasis in different types of cancer. Different studies reported that PDGF-D expression increases in prostate, lung, kidney, ovary, brain, pancreas and especially breast cancer [10, 11-14]. There are limited publications about PDGF-D and molecular mechanism of inducing tumor growth of PDGF-D is not clear [15]. It is known that PDGF-D affect cell invasion, angiogenesis and metastasis and also regulate VEGF signal in different cancer types. Another important effect of PDGF-D on tumor growth and metastasis is having role in epithelialmesenchymal transmission (EMT) [16].

RNA interference (RNAi) is a sequence-specific, posttranscriptional gene regulation mechanism that is based on double-stranded RNA (shRNA, siRNA, miRNA) and respect to these properties it is a new potential therapeutic group $[17,18]$. On the other hand, because of rapid degradation by nucleases, biological instability, inefficient cellular uptake and off-target effects, clinical use of RNAi molecules is limited and an appropriate delivery system is necessary [19$21]$. Even viral vectors frequently are used as delivery system there are several disadvantages like immune reaction and carcinogenic effect and because of these problems, nonviral vectors are gaining popularity as delivery system. Cationic polymers are less toxic compared to liposomes [22]. Chitosan, in addition to its cationic characteristic, is a biodegradable, biocompatible, less immunogenic and safe biopolymer. In spite of viral vectors have high transduction efficiency, they have serious problems such as potential mutation, recombination and oncogenic effects and beside this lipid-based non-viral vectors have also serious problems like toxicity and stability $[23,24]$.

Chitosan, deacetylated form of chitin, is a natural polysaccharide and a proper delivery system [25-31]. Molecular weight and deacetylation degree of chitosan are important for shRNA delivery. In the previous study, our group investigated effect of VEGF targeted shRNA on breast cancer [31]. Xu et al., [32] researched tumor growth and lung metastasis in human kidney carcinoma cell line (SN12-C) by inhibition of PDGF-D with shRNA and observed that this can be a new therapy approach.

In summary, RNAi based therapeutics are promising for breast cancer therapy and it is known that expression of different growth factors increase in breast cancer metastasis. In this in vitro based study, shRNA targeting PDGF-D gene silencing, was encapsulated into chitosan nanoparticles and transfection efficiency of these nanoparticular system was investigated in three different breast cancer cell lines (MCF7, MDA-MB-231 and MDA-MB-435).

\section{Results and Discussion}

The purity, topological forms and accuracy of restriction map of isolated plasmid DNA was determined by agarose gel electrophoresis. As seen in Figure 1, shRNAs are in 95-100\% supercoiled form. After pst1 restriction enzyme application, shRNA forms are given in Figure 2. The purity was checked spectrophotometrically and 260/280 absorbance rate value was found 1.85 .

As mentioned above 5 different shRNA plasmids targeting different exons of PDGF-D gene were used for nanoparticle preparation (NP1-5). Also, mix of them were used for comparison (NPK1-4). Single and multiple PDGF-D

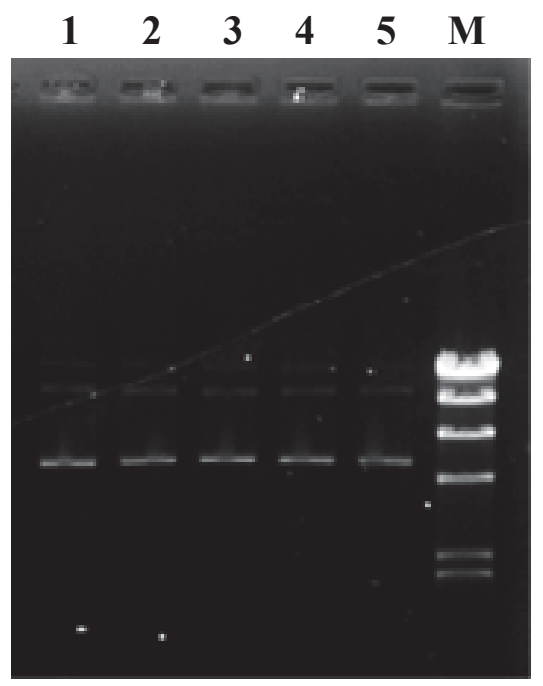

Figure 1. Agarose gel electrophoresis shRNA vectors [1PDGF-D shRNA-1 (sh-1), 2- PDGF-D shRNA-2 (sh-2), 3- PDGF-D shRNA-3 (sh-3), 4- PDGF-D shRNA-4 (sh-4), 5- PDGF-D shRNA-5 (sh-5), M- Lambda DNA/Hind III marker] 


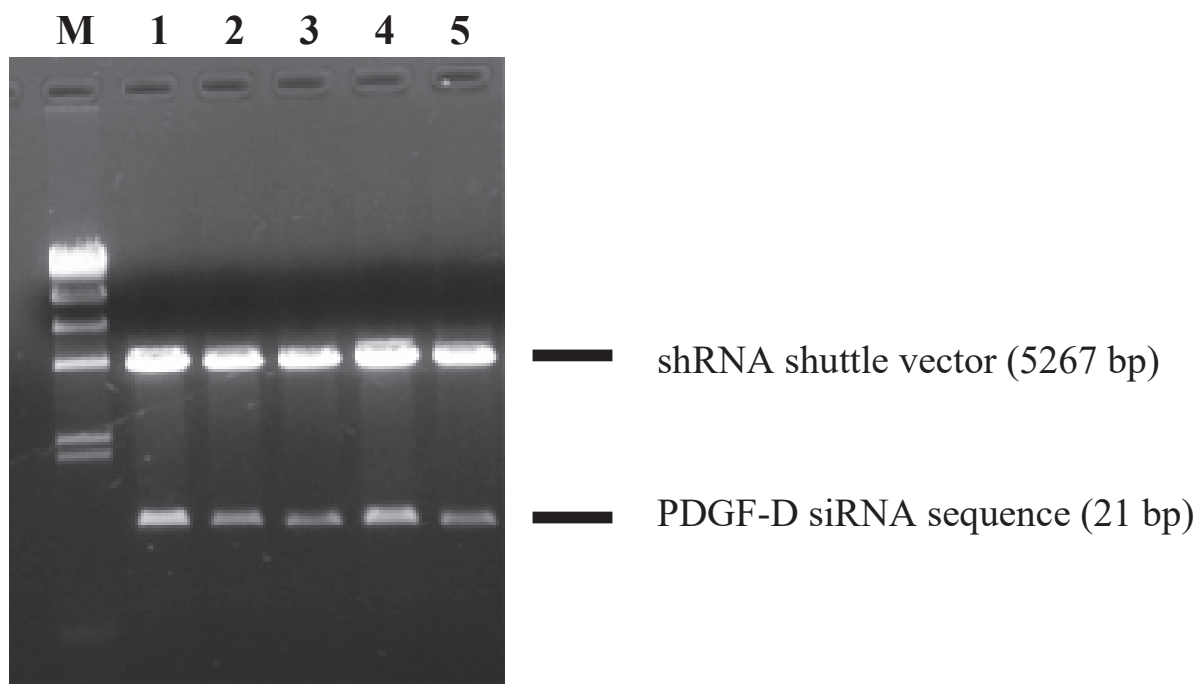

Figure 2. Agarose gel shRNA vectors restriction control [1- PDGF-D shRNA-1 (sh-1), 2- PDGF-D shRNA-2 (sh-2), 3- PDGF-D shRNA-3 (sh-3), 4- PDGF-D shRNA-4 (sh-4), 5- PDGF-D shRNA-5 (sh-5), MLambda DNA/Hind III marker] targeted shRNA sequences were encapsulated in chitosan nanoparticles prepared with different molecular weight chitosan (NP1-5 and NPK1-2 with MMWC, NPK3-4 with LMWC). Two different plasmid amount were used in nanoparticle preparation (NP1-5 and NPK3 with 100 $\mu \mathrm{g}$ DNA, NPK2 and NPK4 with $500 \mu \mathrm{g}$ DNA). Shape of nanoparticles and their surface morphology were shown in Figure 3. Nanoparticles have porous surface as seen in SEM photography.

The characteristics of shPDGF-D loaded CS NPs (encapsulation efficiency, size, zeta potential) were given in Table 1. Encapsulation efficiency of shPDGF-D containing chitosan nanoparticles were about $95.82 \pm 1.07-100 \%$. The particle size of nanoparticles containing single sequence (NP1-NP5) changed between $203.5 \pm 1.5 \mathrm{~nm}$ and $406.1 \pm 15.9$ $\mathrm{nm}$ and particle size of nanoparticles containing multiple sequences (NPK1-NPK4) was $256.1 \pm 0.9 \mathrm{~nm}-400.4 \pm 10.4 \mathrm{~nm}$ $(\mathrm{p}<0.05)$. The surface charge of all formulations was about neutral and zeta potential values were between $1.04 \pm 0.09$ $\mathrm{mV}$ and $5.03 \pm 0.35 \mathrm{mV}$.

To determine whether the chitosan nanoparticles efficiently protected the shRNA from serum degradation, samples was applied agarose gel electrophoresis after serum application. It observed that chitosan nanoparticles protect shPDGF-D from serum during 96 hours (Figure 4).

Release of shRNA from nanoparticles prepared with different sequences was observed during 168 hours. Release profile of nanoparticles with different sequences did not change ( $>0.5)$ (Figure 5).

Release of shRNA from nanoparticles prepared with low molecular weight chitosan (NPK3, NPK4) was faster than nanoparticles prepared with medium molecular weight chitosan (NP1-5, NPK1, NPK2). shRNA release from
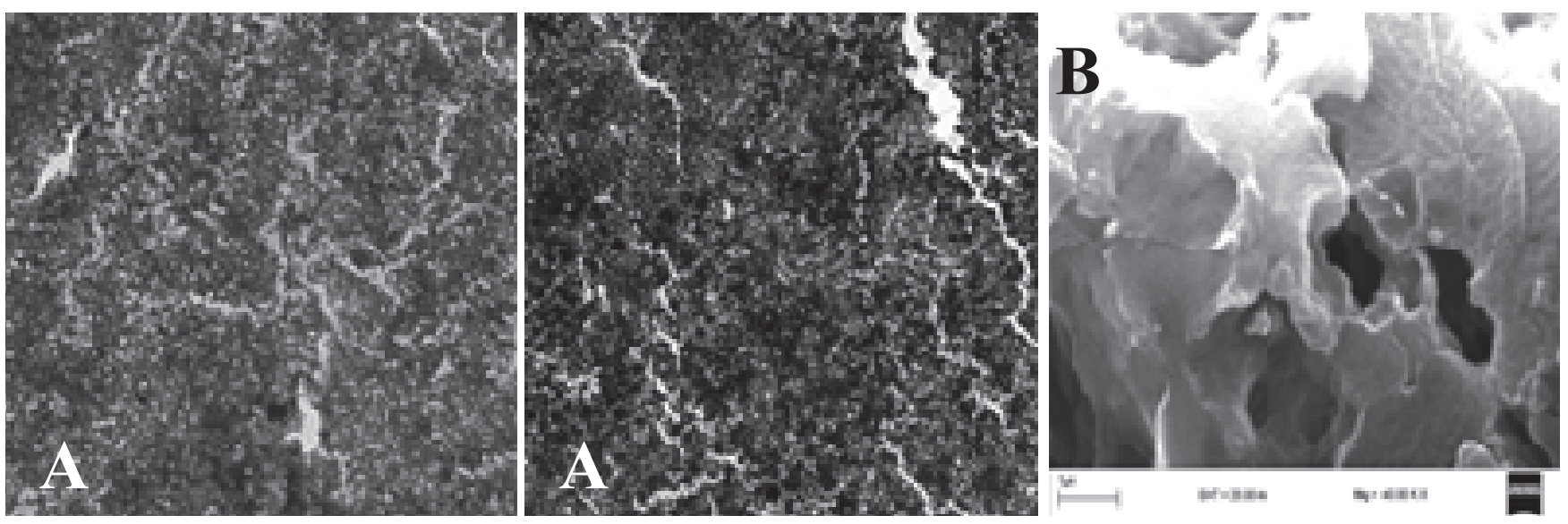

Figure 3. A; Transmission (TEM) and B; Scanning (SEM) electromicrographs of shRNA-CS NPs. 
Table 1. Encapsulation efficiency, zeta potential and size values of shRNA loaded chitosan nanoparticles (shRNA-CS NPs).

\begin{tabular}{|l|c|c|c|}
\hline \multicolumn{1}{|c|}{ Codes } & $\begin{array}{c}\text { Encapsulation efficiency } \\
(\% \pm \text { SD) }\end{array}$ & $\begin{array}{c}\text { Zeta Potential } \\
(\mathbf{m V} \pm \text { SD) }\end{array}$ & $\begin{array}{c}\text { Particle Size } \\
(\mathbf{n m} \pm \text { SD })\end{array}$ \\
\hline shRNA & - & $-19.85 \pm 0.1$ & - \\
\hline Chitosan & - & $28.45 \pm 0.1$ & $247.5 \pm 16.5$ \\
\hline NP1 & $100.0 \pm 5.1$ & $5.03 \pm 0.4$ & $341.7 \pm 2.8$ \\
\hline NP2 & $100.0 \pm 2.4$ & $5.29 \pm 0.1$ & $203.5 \pm 1.5$ \\
\hline NP3 & $100.0 \pm 2.0$ & $2.61 \pm 0.5$ & $311.3 \pm 3.1$ \\
\hline NP4 & $95.82 \pm 1.1$ & $1.94 \pm 1.2$ & $406.1 \pm 15$. \\
\hline NP5 & $97.44 \pm 1.1$ & $1.04 \pm 0.1$ & $256.1 \pm 0.9$ \\
\hline NPK1 & $97.44 \pm 0.8$ & $1.15 \pm 0.1$ & $295.6 \pm 1.2$ \\
\hline NPK2 & $99.20 \pm 4.0$ & $1.87 \pm 0.1$ & $400.4 \pm 10.4$ \\
\hline NPK3 & $96.09 \pm 0.2$ & $1.24 \pm 0.1$ & $308.2 \pm 4.9$ \\
\hline NPK4 & $98.57 \pm 3.6$ & $1.27 \pm 0.1$ & \\
\hline
\end{tabular}

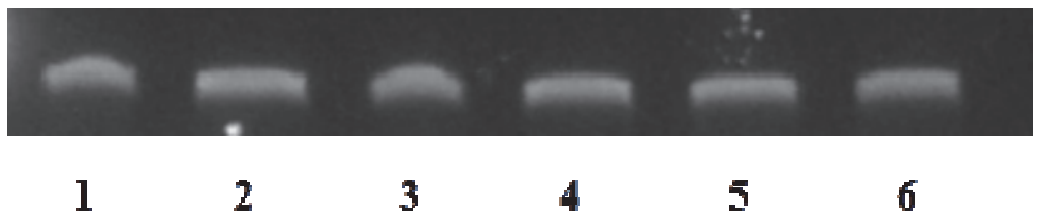

Figure 4. Serum stability of shRNA-CS NPs (Lane 1; initial, lanes 2-6; after 4, 24, 48, 72 and 96 hours)

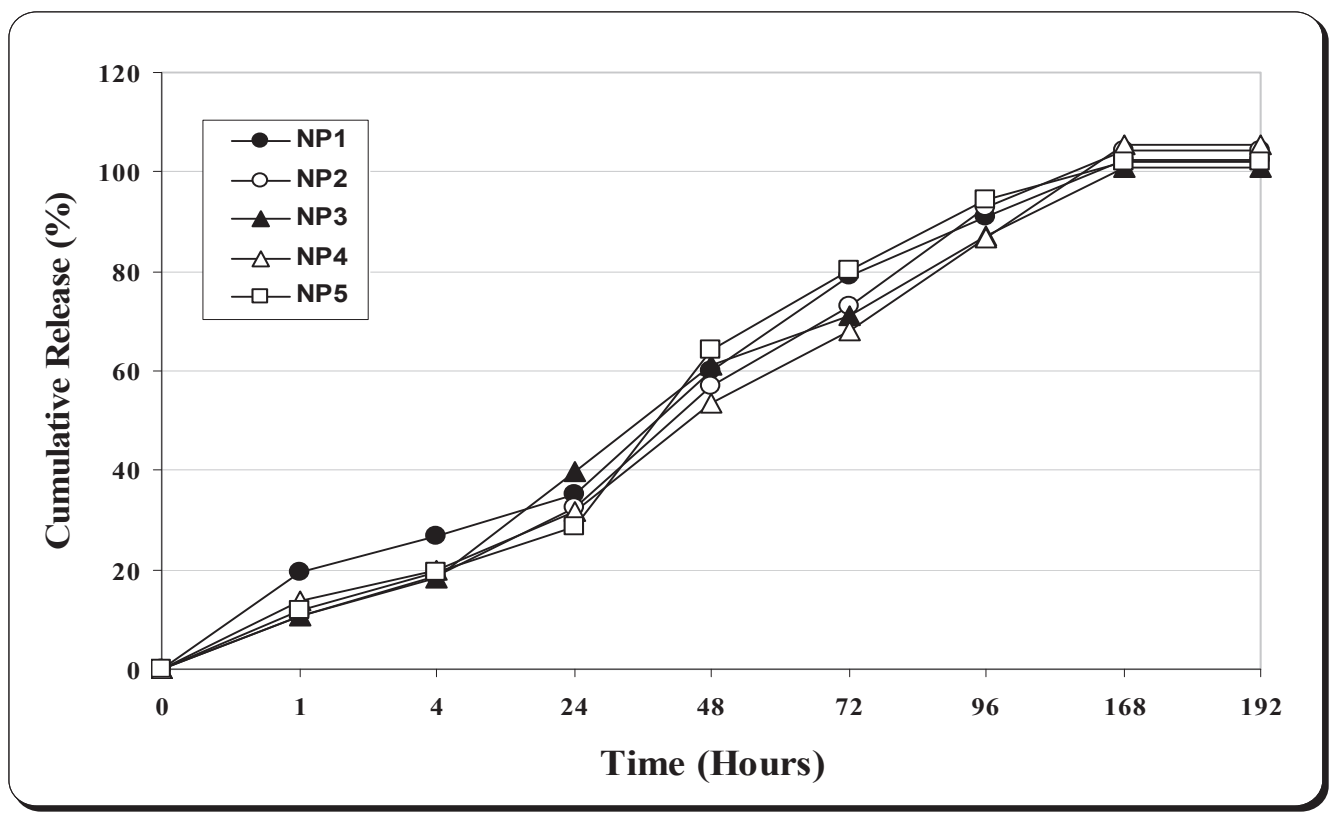

Figure 5. Release profiles of PDGF-D shRNA from CS NPs. 
nanoparticles prepared with low molecular weight chitosan completed in 96 hours and release from nanoparticles prepared with medium molecular weight chitosan was observed during 168 hours (Figure 6).

Release profiles of nanoparticles prepared with different amount of shRNA (100-500 $\mu \mathrm{g})$ and single/multiple sequences did not change $(\mathrm{p}>0.05)$ (Figure 7$)$. By these results, while molecular weight of chitosan has an effect on release profile of nanoparticles, shRNA amount and different sequences have not.

To determine effect of encapsulation process and release studies on plasmid integrity, agarose gel electrophoresis was applied to the samples after release studies. As shown in Figure 8 shRNA remained stable during nanoparticle preparation procedure, centrifugation, lyophilisation and in vitro release studies. This result showed similarity with our earlier studies.

Cellular uptake of shPDGF-D/chitosan nanoparticles controlled with GFP marker gene which is encoded by shRNA plasmid vector. To determine cellular uptake and transfection efficiency, GFP expression was observed by fluorescence microscopy. As shown in Figures 9 and 10, cells were transfected successfully and $80-85 \%$ transfection efficiency was observed and GFP expression increased with time.

Three different breast cancer cell lines (MCF-7, MDA-MB-231 and MDA-MB-435) were used for in vitro transfection studies and PDGF-D amount after transfection was measured in cell homogenate using ELISA. Endogen PDGF-D expression and gene silencing efficiency at 3 different cell lines were shown in Figure 11. As shown in Figure 11, PDGF-D expression reduced in each breast cancer cell line at 168 hours after transfection. PDGF-D silencing values changed between 69.17-82.20\% in MCF-7, 47.5-92.14\% in MDA-MB-231 and 10.44-88.44\% in MDA-MB-435 after 168 hours. Either cell line type or nanoparticles with different sequences affect the knock down of PDGF-D gene level. Different silencing efficiency was also observed with nanoparticles containing single and multiple sequences of shRNA. In MCF-7, silencing effect of formulations prepared with single sequences (NP1-NP5) was between 69.17 and $80.87 \%$ and difference between NP4, NP5 and NP1, NP2, NP3 was significant particularly $(\mathrm{p}<0.05)$ (Figure 11). In MDA-MB-231 cell line, a significant difference was found between NP1 and other formulations $(\mathrm{p}<0.05)$ (Figure 11). However silencing difference between formulations containing single sequences (NP1-NP5) was not significant in MDA-MB-435 cell line $(\mathrm{p}>0.05)$ (Figure 11).

When a comparison was made between formulations containing single and multiple sequences, a significant difference was observed between NPK1 and other formulations except NP4 and NP5 in MCF-7 cell line ( $\mathrm{p}<0.05)$ (Figure 11). Different gene silencing effect in MDA-MB-231 was highly significant $(\mathrm{p}<0.001)$ and in MDA-MB-435 while difference between NP3, NP4 and NPK1 was highly

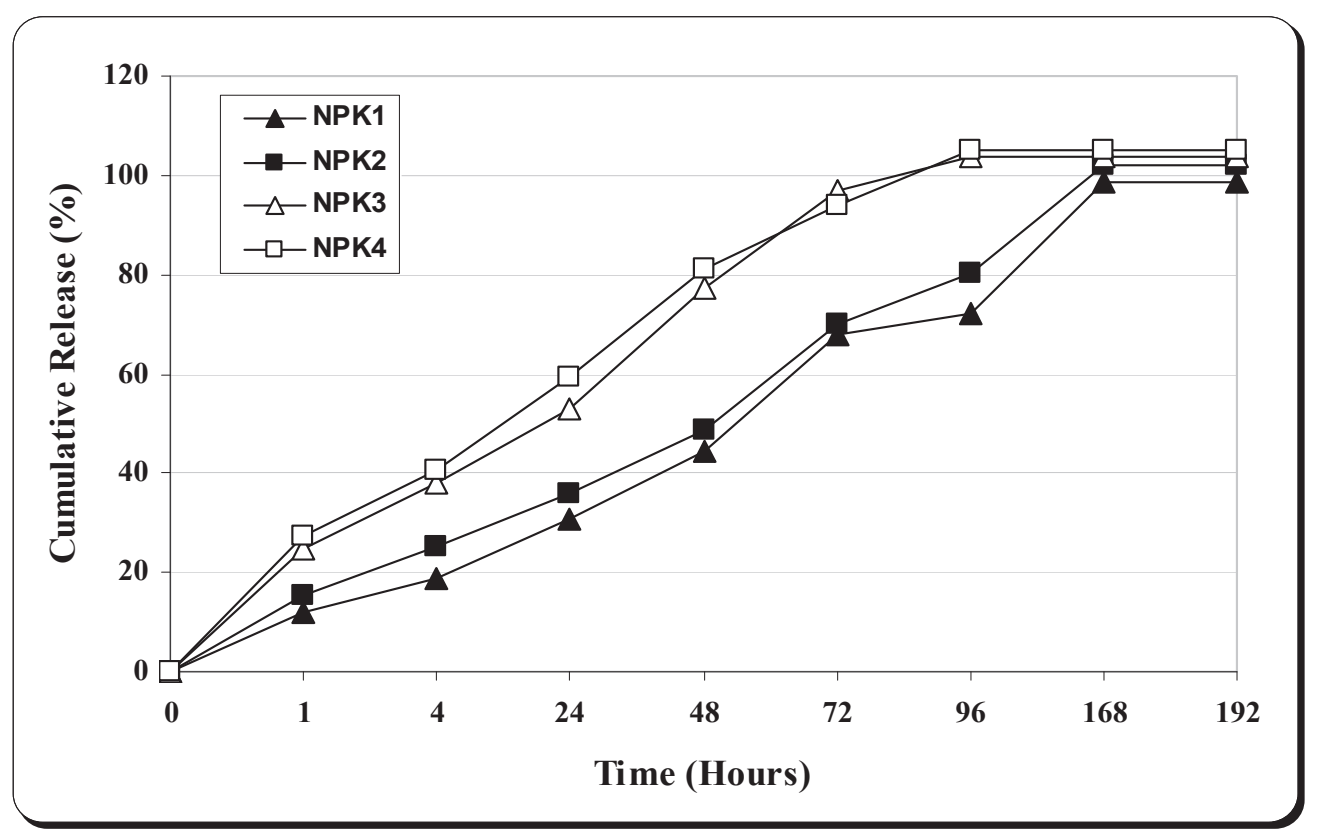

Figure 6. Effect of molecular weight of chitosan on release profiles of shRNA CS NPs. 


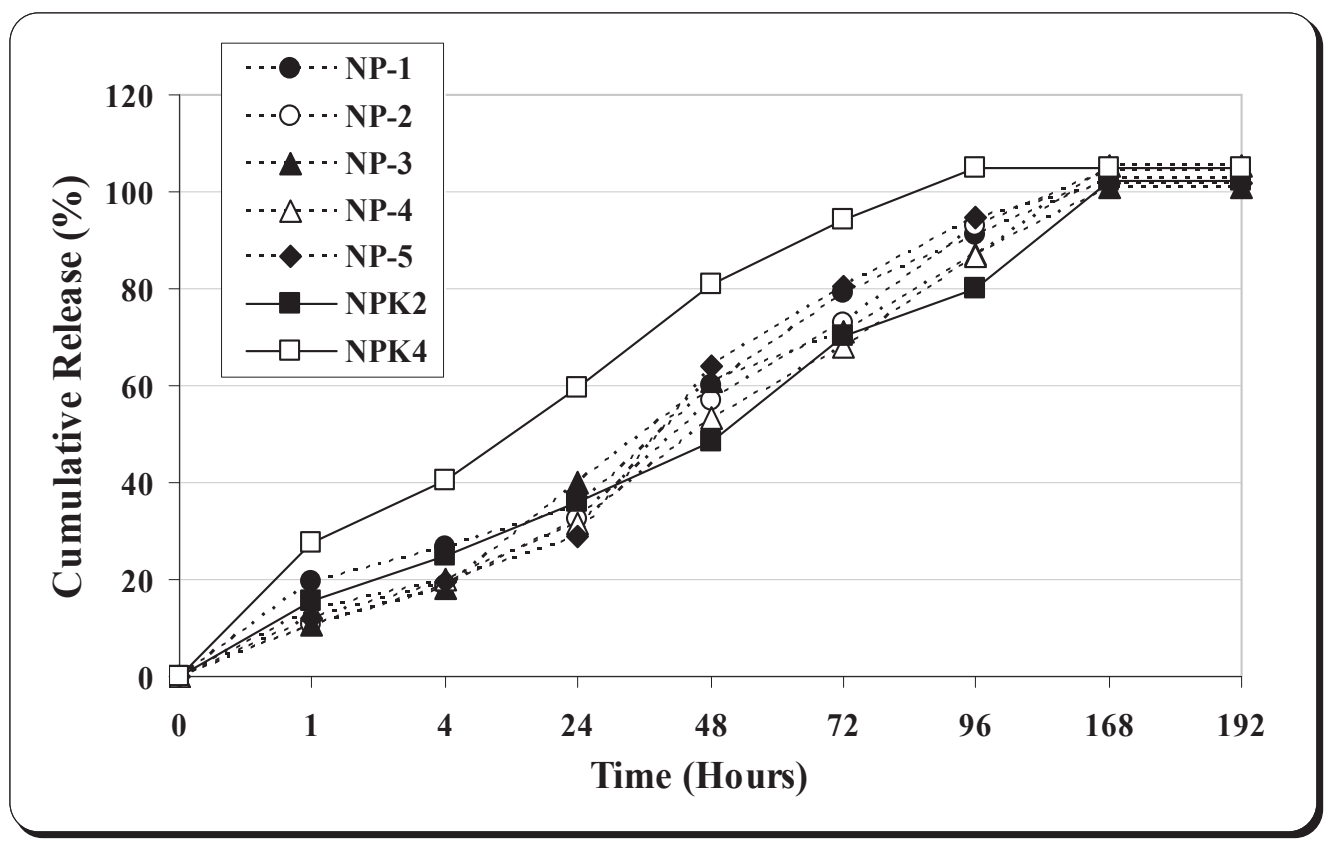

Figure 7. Effect of shRNA amount on release profiles of shRNA CS NPs.

significant $(\mathrm{p}<0.001)$, difference between other formulations and NPK1 was significant $(\mathrm{p}<0.05)$ (Figure 11).

When gene silencing effect of formulations containing different molecular weight chitosan (NPK1/NPK3 or NPK2/ NPK4) compared, a highly significant difference was found at PDGF-D inhibition level in each 3 cell lines $(\mathrm{p}<0.001)$ (Figure 11).

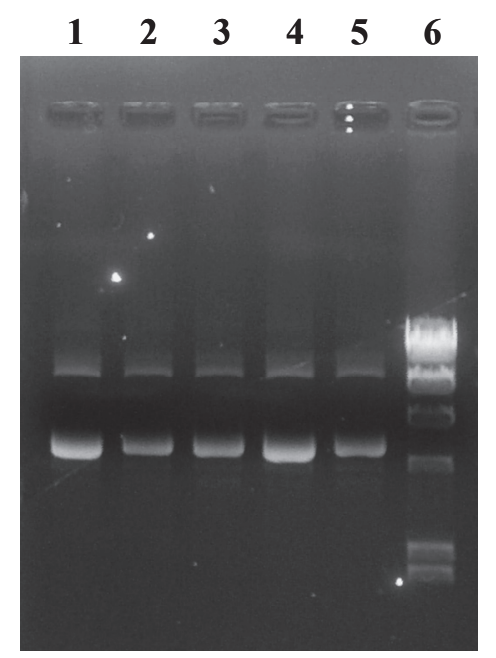

Figure 8. Agarose gel shRNA after release studies. (Lane 1, NP5; Lane 2, NP4; Lane 3, NP3; Lane 4, NP2; Lane 5, NP1; Lane 6, marker)
Effect of shRNA amount (NPK1-NPK4) on PDGF-D inhibition in MCF-7 cell line was not significant ( $p>0.05$ ) (Figure 11). In MDA-MB-231, shRNA amount affected PDGF-D silencing in nanoparticles only containing low molecular weight chitosan $(\mathrm{p}<0.05)$ and in MDA-MB-435 cell line, shRNA amount affected silencing in formulations only containing medium chitosan $(\mathrm{p}<0.05)$ (Figure 11).

MDA-MB-231 cell line has the highest endogen PDGF-D amount while MDA-MB-435 cell line has the lowest. When the PDGF-D inhibition effect of nanoparticles compared at different breast cancer cell lines it showed the following rank (MDA-MB-231>MCF-7>MDA-MB-435) (Figure 11). Based on this, MDA-MB-231 cell line used in gene silencing studies at different time intervals trying to find the time dependent effect of gene inhibition (Figure 12). As shown in Figure 12, shRNA-CS NPs showed PDGF-D gene silencing affect in a time-dependent manner. While silencing of single sequence containing formulations (NP1-NP5) was $53.65-75.11 \%$ at 72 hours, silencing effect of NP1-NP5 was between 92.14 and $84.68 \%$ at 168 hours $(\mathrm{p}<0.05)$. NP1 had the highest silencing effect at 168 hours in MDA-MB-231 cell line and difference between NP1 and other formulations was statistically significant $(\mathrm{p}<0.05)$ (Figure 12). Formulations prepared with different single sequences (NP1-NP5) had higher silencing effect than 

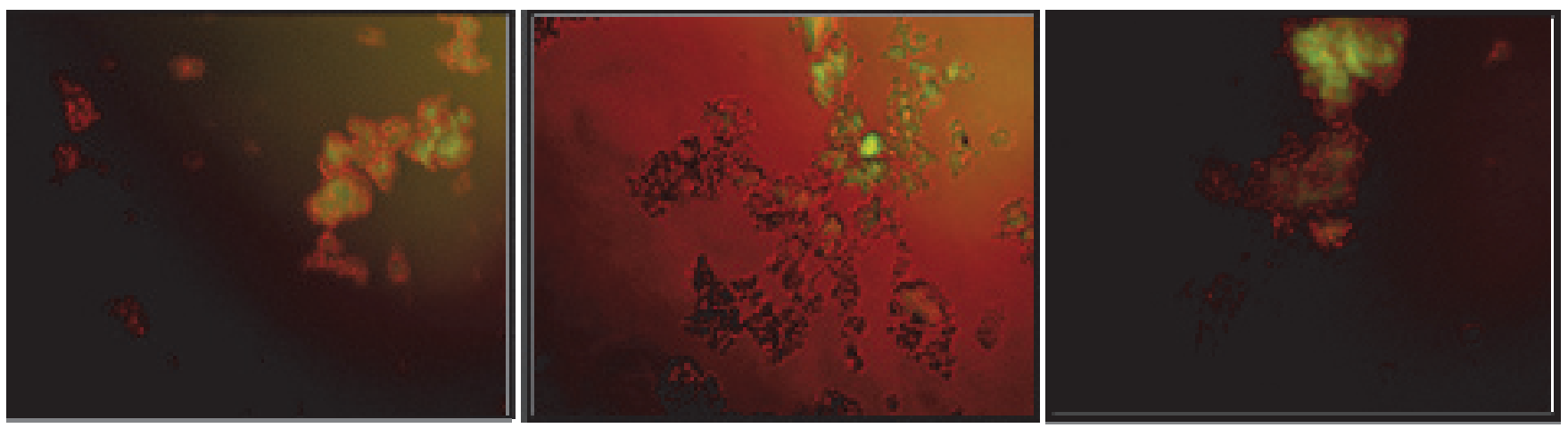

Figure 9. Cellular uptake of shRNA CS NPs 24 hours after transfection
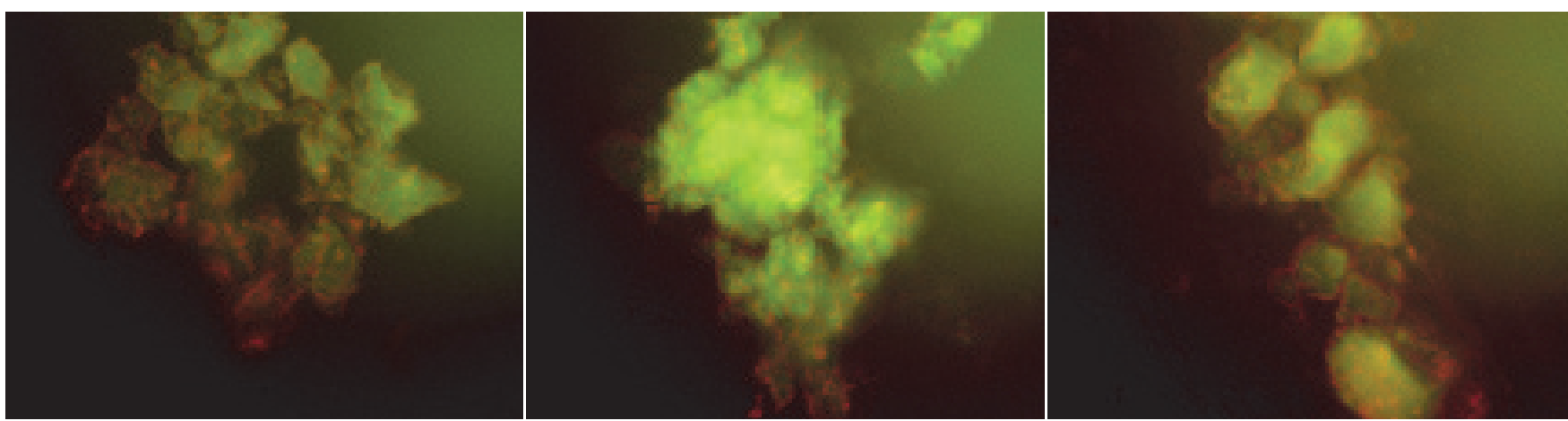

Figure 10. Cellular uptake of shRNA CS NPs 48 hours after transfection

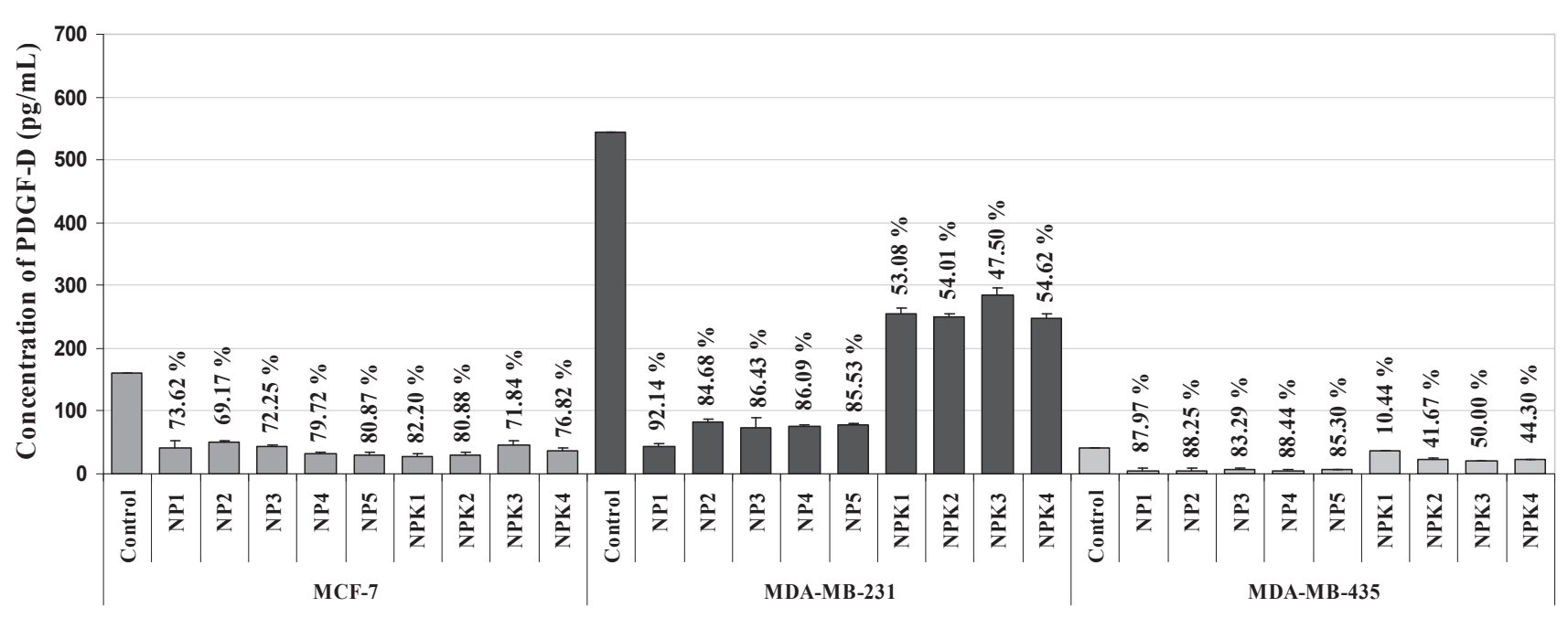

Figure 11. PDGF-D silencing effect of shRNA-CS NPs 168 hours after transfection at different breast cancer cell lines. 


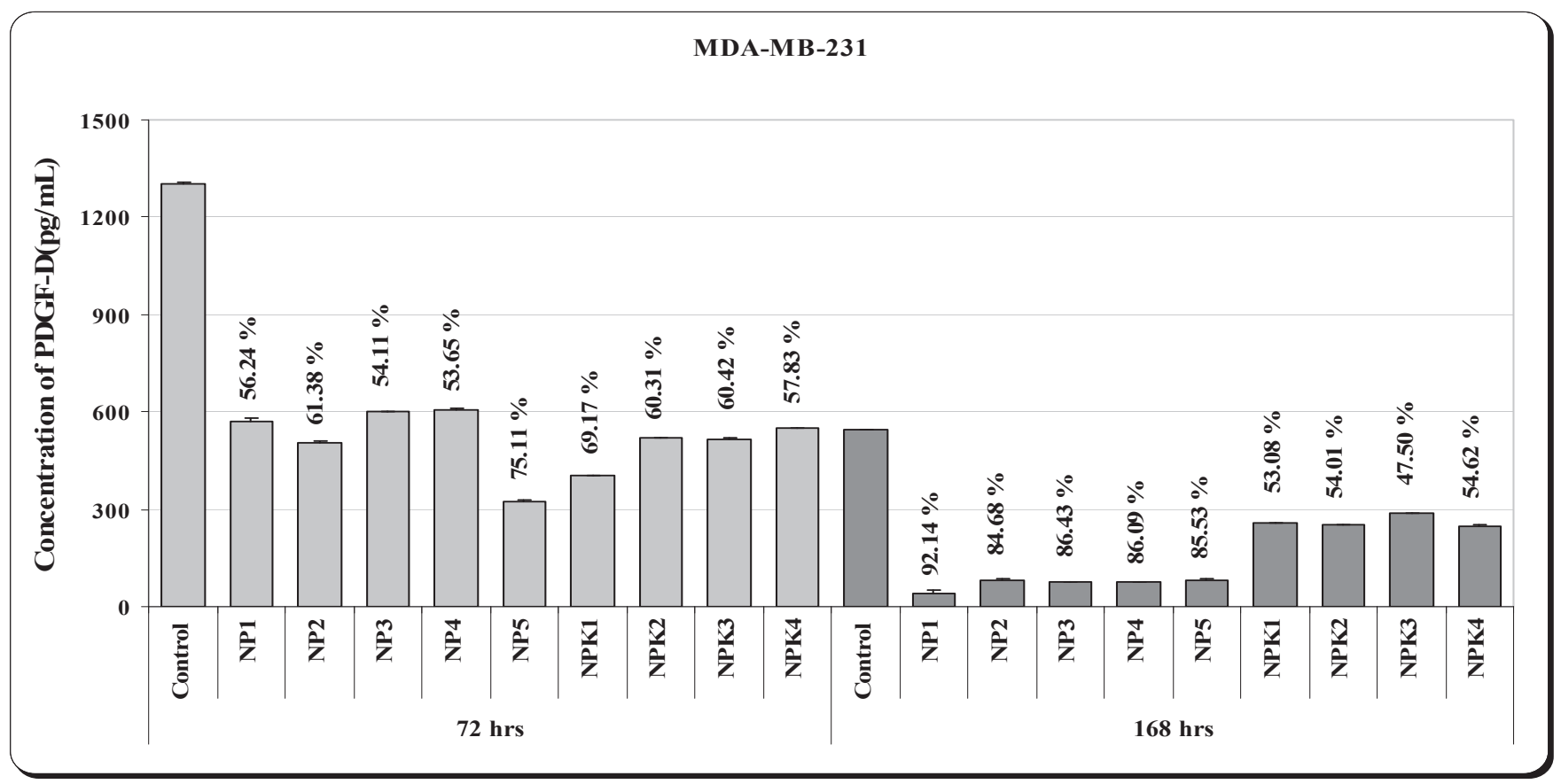

Figure 12. Time-dependent PDGF-D inhibition effect of shRNA-CS NPs in MDA-MB-231 cell line.

formulations prepared with multiple sequences (NPK1NPK4) and difference between formulations prepared with different single sequences was significant $(\mathrm{p}<0.05)$ (Figure 12). When effect of shRNA amount in silencing efficiency investigated, difference between formulations prepared with medium molecular weight chitosan was not significant ( $p>0.05)$ while difference between formulations prepared with low molecular weight chitosan was significant $(\mathrm{p}<0.05)$.

We concluded that difference between silencing efficiency of formulation related to plasmids that they contain targeting different exons of PDGF-D gene and formulation containing plasmid targeting exon 6 of PDGF-D gene (NP1) has the highest silencing efficiency. These results have similarity with study carried out with siRNA VEGF targeting different isoforms of VEGF [36].

\section{Conclusions}

In this study, 5 plasmids targeting different exon regions of PDGF-D gene were used and it was obtained that formulation containing shRNA plasmid targeting PDGF-D exon 6 (NP1) has the highest silencing efficiency. It was also evaluated that chitosan can be a suitable gene delivery system for shRNA targeting PDGF-D.

\section{Materials and Methods}

\subsection{Cell lines and reagents}

Three different breast cancer cell lines MCF-7, MDA-MB-231 and MDA-MB-435 were obtained from American Type Culture Collection (ATCC). Cell lines were cultured in Dulbecco's minimum essential medium (DMEM) (Biological Industries, Israel) containing 10\% fetal bovine serum (FBS) (Biological Industries, Israel), $100 \mathrm{mM}$ glutamin and 100 $\mathrm{mM}$ antibiotics solution. Two types of chitosan with different molecular weight (low molecular weight, $150 \mathrm{kDa}, \mathrm{DD} 93$; medium molecular weight, $400 \mathrm{kDa}, \mathrm{DD}$ 87) were purchased from Fluka, Germany. Human PDGF-D ELİSA kit (USCN Life Science, USA) was used in determination of PDGF-D level. All other chemicals and reagents were of molecular grade and purchased from Sigma-Aldrich.

\subsection{PDGF-D shRNA plasmid}

pGeneClip hMGFP expression vector, encodes human PDGF-D, contains CMV and humanU1 promoter and GFP and ampicillin selection marker genes (5.2 kb) (SureSilencing KH00048G, Qiagen, USA). Studies were carried out with five different shRNA plasmids targeting different exons of PDGF-D gene. The plasmids were transformed into Esherichia coli DH5a bacterial strain by using classical $\mathrm{CaCl}_{2}$ method and isolated by the alkaline-lysis technique [33]. 


\subsection{Spectrophotometric and electrophoretic controls of ShRNA}

The quantity and quality of isolated plasmid DNA were determined by spectrophotometrically at 260 and $280 \mathrm{~nm}$ (Shimadzu UV-Biospec 1601, Japan). The purity and forms of plasmid DNA was analyzed by electrophoresis in agarose gel. For this purpose, plasmid DNA was applied into 0.8\% agarose gel containing tris-boric acid-EDTA (TBE, pH 8.0) and ethidium bromide at constant voltage $(80 \mathrm{~mA}, 200 \mathrm{~V})$ during 1 hour. Conformation of DNA was checked using DC290 Camera and 1D Image Analysis Software system (Kodak, USA) under UV light. The percentage of supercoiled DNA was determined by using following equation [34]:

$\left.\mathrm{SC}(\%)=\mathrm{V}_{\mathrm{SC}}-\mathrm{V}_{0} /\left[\mathrm{V}_{\mathrm{SC}}+\mathrm{V}_{\mathrm{L}}+\mathrm{V}_{\mathrm{OC}}\right)-\left(3 \times \mathrm{V}_{0}\right)\right]$

$\left(\mathrm{V}_{\mathrm{SC}}\right.$, volume of supercoiled DNA; $\mathrm{V}_{\mathrm{L}}$, volume of lineer DNA; $\mathrm{V}_{\mathrm{OC}}$, volume of open

Circular DNA; $\mathrm{V}_{0}$, volume of background)

\subsection{Preparation of shRNA loaded nanoparticles}

shRNA loaded chitosan nanoparticles were prepared by ionic gelation method as previously described [35]. Briefly, tripolyphosphate solution $(0.25 \% \mathrm{w} / \mathrm{v})$ containing plasmid dropped into chitosan solution $(0.25 \% \mathrm{w} / \mathrm{v})$ under constant stirring. After one hour stirring, nanoparticles were separated by centrifugation at $12.000 x g$ for 15 minutes. The supernatant was discarded and particles were washed with bidistilled water three times. Nanoparticles were stored at $+4^{\circ} \mathrm{C}$ after freeze-drying. In order to study effect of formulation variables [molecular weight of chitosan (150 and $400 \mathrm{kDa}$ ), plasmid type (5 different plasmid and mix of these) and plasmid concentration (100 and $500 \mu \mathrm{g})$ ] different nanoparticle formulations were prepared (Table 2).

\subsection{Characterization of nanoparticles}

The morphological examination and size control of nanoparticles were performed by transmission electron microscopy (TEM) (JEM 1200 EXII, Jeol, Japan) and scanning electron microscopy (SEM) (JSM-5910 LV, Jeol, Japan).

Encapsulation efficiency, the amount of DNA encapsulated in nanoparticles, was calculated by measuring the difference between the total DNA amount added in nanoparticle solution and the amount of not-entrapped DNA remaining in the supernatant after centrifugation. For this purpose, the supernatant after centrifugation was measured spectrophotometrically at 260 and $280 \mathrm{~nm}$.

Measurements of size and zeta potential of nanoparticles were performed by Zetasizer (NanoZS ZEN3500, Malvern Instrumentals Ltd., UK). The samples were measured in phosphate saline buffer (PBS pH 7.4) and each measurement was done in triplicate.

\subsection{Serum stability of nanoparticles}

Stability of shRNA-chitosan nanoparticles against serum was studied by incubating samples in PBS containing 10\% inactivated fetal bovine serum at $37^{\circ} \mathrm{C}$. Samples were taken at different time intervals. The integrity of shRNA was examined by using agarose gel electrophoresis.

\subsection{In vitro release studies}

The release of shRNA from nanoparticles was determined in shaking water bath at $37^{\circ} \mathrm{C}$. For in vitro release studies, nanoparticles containing $7 \mu \mathrm{g}$ shRNA were suspended in $2 \mathrm{ml}$ PBS (pH 7.4) and incubated at $37^{\circ} \mathrm{C}$. Samples were removed at different time intervals and centrifuged for 15 minutes at $14.000 \mathrm{~g}$ and the release medium was replaced with fresh

Table 2. Composition of shRNA loaded chitosan nanoparticles (shRNA-CS NPs).

\begin{tabular}{|c|c|c|c|}
\hline Codes & PDGF-D shRNA* & $\begin{array}{c}\text { Chitosan } \\
\text { Mol wt / Conc. (\%) }\end{array}$ & $\begin{array}{c}\text { Amount } \\
\text { of shRNA }(\mu \mathrm{g})\end{array}$ \\
\hline NP1 & 1 & \multirow{7}{*}{$400 / 0.25$} & \multirow{6}{*}{100} \\
\hline NP2 & 2 & & \\
\hline NP3 & 3 & & \\
\hline NP4 & 4 & & \\
\hline NP5 & 5 & & \\
\hline NPK1 & \multirow{4}{*}{$1-5$} & & \\
\hline NPK2 & & & 500 \\
\hline NPK3 & & \multirow{2}{*}{$150 / 0.25$} & 100 \\
\hline NPK4 & & & 500 \\
\hline
\end{tabular}


buffer. The amount of shRNA released in the supernatant was measured spectrophotometrically at $260 \mathrm{~nm}$. Additionally, the structural integrity of released shRNA from nanoparticles was controlled with agarose gel electrophoresis.

\subsection{In vitro transfection studies}

In vitro transfection studies were performed in MCF7, MDA-MB-231 and MDA-MB-435 cell lines. The cells were cultured in DMEM containing 10\% FBS, $100 \mathrm{mM}$ L-glutamine and $100 \mathrm{mM}$ antibiotic solutions and maintained at $37^{\circ} \mathrm{C}$ in a humidified $5 \% \mathrm{CO}_{2}$ incubator. For transfection studies, the cells were seeded in 24 -well plate at a density 2 x $10^{4}$ cells/well and incubated overnight. Transfection was performed on cells that were approximately $70 \%$ confluent. shRNA loaded nanoparticles were suspended in the serumfree medium and added to the cells ( $5 \mu \mathrm{g}$ shRNA/well). The cells were incubated for 4 hours at incubator and then serumcontaining fresh medium was added to the wells. Cells were incubated at incubator for 7 days.

\subsection{Cellular uptake of nanoparticles}

In order to investigate the cellular uptake of PDGF-D targeting shRNA loaded nanoparticles, cells were observed with GFP marker gene encoded by shRNA plasmid by using fluorescence microscopy at different time intervals.

\subsection{Determination of PDGF-D levels in cells by ELISA}

Three and seven days after transfection the medium was discarded and the cells were washed with PBS. Then the cells were lysed in $200 \mu$ l Ripa Buffer by 3 freezing and thawing cycles. The expression of PDGF-D was determined in cell lysate by ELİSA kit according to manufacturer's instructions and read spectrophotometrically at $450 \mathrm{~nm}$.

\subsection{Statistical analysis}

All results related to in vitro studies were evaluated and differences were compared using SPSS-12. Statistical significance was determined when $\mathrm{p}<0.05$.

\section{Acknowledgement}

This study was supported by The Scientific and Technological Research Council of Turkey (TUBITAK), grant no:113S003 and this study was also presented as a poster in ESGCT
Congress in 23-26 October 2014, The Hague, Holland and the poster abstracts were published in Human Gene Therapy.

\section{Authorship statement}

Author contributions: Concept - J.A., S.Ö.T.; Design - J.A., S.Ö.T., C.E.; Supervision - J.A.; Resource - J.A., S.Ö.T.; Materials - J.A., S.Ö.T.; Data Collection and/or Processing S.Ö.T, C.E.; Analysis and/or Interpretation - J.A., S.Ö.T., C.E.; Literature Search - J.A., S.Ö.T., C.E.; Writing - C.E., J.A.; Critical Reviews - J.A., S.Ö.T., C.E.

\section{Conflict of interest statement}

The authors declared no conflict of interest.

\section{References}

1. Rahman MA, Toi M. Anti-angiogenic therapy. Biomed Pharmacother 2003; 57: 463-70.

2. Raica M, Cimpean AM. Platelet-derived growth factor (PDGF)/PDGF receptors (PDGFR) axis as target for antitumor and antiangiogenic therapy. Pharmaceuticals 2010; 3: 572-99.

3. Heldin C, Westermark B. Mechanism of action and in vivo role of platelet-derived growth factor. Phys Rev 1999; 79: 1283-316.

4. Demoulin J, Essaqhir A. PDGF receptor signaling networks in normal and cancer cells. Cytokine Growth Factor Rev 2014; 25: 273-83.

5. Bergsten E, Uutela M, Li X. PDGF-D is a specific, proteaseactivated ligand for the PDGF beta-receptor. Nat Cell Biol 2001; 3: 512-6.

6. LaRochelle WJ, Jeffers M, McDonald WF. PDGF-D, a new protease-activated growth factor. Nat Cell Biol 2001; 3: 51721.

7. Li X, Eriksson U. Novel PDGF family members: PDGF-C and PDGF-D. Cytokine Growth Factor Rev 2003; 14: 91-8.

8. Rosenkranz S, Kazlauskas A. Evidence for distinct biological properties and signaling machineries of the PDGF receptor a and b subtypes. Growth Factors 1999; 16: 201-16.

9. Yu J, Ustach C, Kim HR. Platelet-derived growth factor signaling and human cancer. J Biochem Mol Biol 2003; 36: 4959.

10. Wang Z, Kong D, Li Y, Sarkar FH. PDGF-D signaling: a novel target in cancer therapy. Curr Drug Targets 2009; 10: 38-41.

11. Ustach CV, Taube ME, Hurst NJ Jr, Bhagat S, Bonfil RD, Cher ML, Schuger L, Kim HR. A potential oncogenic activity of platelet-derived growth factor $\mathrm{d}$ in prostate cancer progression. Cancer Res 2004; 64: 1722-9.

12. Ustach CV, Choi KH-R. Platelet-derived growth factor $\mathrm{d}$ is activated by urokinase plasminogen activator in prostate carcinoma cells. Mol Cell Biol 2005; 25: 6279-88.

13. Wang Z, Kong D, Banerjee S, Li Y, Adsay NV, Abbruzzese J, Sarkar FH. Down-regulation of platelet-derived growth 
factor-D inhibits cell growth and angiogenesis through inactivation of Notch-1 and nuclear factor-kappa B signaling. Cancer Res 2007; 67: 11377-85.

14. Kong D, Wang Z, Sarkar SH, Li Y, Banerjee S, Saliganan A, Kim HR, Cher ML, Sarkar FH. Platelet-derived growth factor-d overexpression contributes to epithelial-mesenchymal transition of PC3 prostate cancer cells. Stem Cells 2008; 26: 1425-35.

15. Wang Z, Ahmad A, Li Y, Kong D, Azmi AS, Banerjee S, Sarkar FH. Emerging roles of PDGF-D signaling pathway in tumor development and progression. Biochimica Biophysica Acta 2010; 1806: 122-30.

16. Devarajan E, Song Y, Krishnappa S, Alt E. Epithelialmesenchymal transition in breast cancer lines is mediated through PDGF-D released by tissue-resident stem cells. Int J Cancer 2012; 131: 1023-31.

17. Whelan J. First clinical data on RNAi. Drug Discov Today 2005; 10: 1014-5.

18. Corey DR. Chemical modification: the key to clinical application of RNA interference? J Clin Invest 2007; 117: 3615-22.

19. Urban-Klein B, Werth S, Abuharbeid S, Czubayko F, Aigner A. RNAi-mediated gene-targeting through systemic application of polyethylenimine (PEI)-complexed siRNA in vivo. Gene Ther 2005; 12: 461-6.

20. Howard KA, Rahbek UL, Liu X, Damgaard CK, Glud SZ, Andersen MØ, Hovgaard MB, Schmitz A, Nyengaard JR, Besenbacher F, Kjems J.RNA intereference in vitro and in vivo using a novel chitosan/siRNA nanoparticle system. Mol Ther 2006; 14: 476-84.

21. Howard KA, Paludan SR, Behlke MA, Besenbacher F, Deleuran B, Kjems J. Chitosan/siRNA nanoparticle-mediated TNF-alpha knockdown in peritoneal macrophages for antiinflammatory treatment in a murine arthritis model. Mol Ther 2009; 17: 162-8.

22. Al-Dosari MS, Gao X. Non-viral gene delivery: principle, limitations, and recent progress. AAPS J 2009; 11: 671-81.

23. Scales CW, Huang F, Li N, Vasilieva YA, Ray J, Convertine AJ, McCormick MC. Corona-stabilized interpolyelectrolyte complexes of siRNA with non-immunogenic, hydrophilic/ cationic block copolymers prepared by aqueous RAFT polymerization. Macromolecules 2006; 39: 6871-81.

24. Filion MC, Phillips NC. Major limitations in the use of cationic liposomes for gene delivery. Int J Pharm 1998; 162: 159-70.

25. Mao H-Q, Roy K, Troung-Le VL, Janes KA, Lin KY, Wang Y, August JT, Leong KW.. Chitosan-DNA nanoparticles as gene carriers: synthesis, characterization and transfection efficiency. J Control Release 2001; 70: 399-421.

26. Akbuğa J, Aral C, Özbaş-Turan S, Kabasakal L, Keyer-Uysal M. Transfection efficiency of chitosan microspheres effect of DNA topology. STP Pharm Sci 2003; 13: 99-103.

27. Ozbas-Turan S, Akbuğa J, Enneli B. Evaluation of antisense oligonucleotide loaded chitosan nanoparticles; characterization and antisense effect. Pharmazie 2009; 64: 807-11.

28. Salva E, Akbuga J. Comparison of silencing effect of chitosan/ psiRNA complexes in different cell lines. Adv Chitin Sci 2009; 11: 203-8.

29. Ozbaş-Turan S, Akbuğa J, Sezer AD. Topical application of antisense oligonucleotide-loaded chitosan nanoparticles to rats. Oligonucleotides 2010; 20: 147-53.

30. Salva $\mathrm{E}$, Akbuğa J. In vitro silencing effect of chitosan nanoplexes containing siRNA expressing vector targeting VEGF in breast cancer cell lines. Pharmazie 2010; 65: 896-903.

31. Salva E, Kabasakal L, Eren F, Çakalağaoğlu F, Özkan N, Akbuğa J. Chitosan/short hairpain RNA complexes for vascular endothelial growth factor suppression invasive breast carcinoma. Oligonucleotides 2010; 20: 183-90.

32. Xu L, Tong R, Cochran DM, Jain RK. Blocking platelet-derived growth factor-D/platelet-derived growth factor receptor beta signaling inhibits human renal cell carcinoma progression in an orthotropic mouse model. Cancer Res 2005; 65: 5711-9.

33. Sambrook J, Fritsch EF, Maniatis T (eds). Molecular Cloning: A Laboratory Manual. Cold Spring Harbor Laboratory Press: USA, 1989.

34. Ando S, Putnam D, Pack DW, Langer R. PLGA microspheres containing plasmid DNA: Preservation of supercoiled DNA via cryopreparation and carbohydrate stabilization. J Pharm Sci 1999; 88: 126-30.

35. Calvo P, Remunan-Lopez C, Vila-Jato JL, Alonso MJ. Novel hydrophilic chitosan-polyethylene oxide nanoparticles as protein carriers. J Appl Polym Sci 1997; 63: 125-32.

36. Zhang L, Yang L, Mohamed-Hadley A, Rubin S.C, Coukosa G. Vector-based RNAi, a novel tool for isoform-specific knockdown of VEGF and anti-angiogenesis gene therapy of cancer. Biochem Biophys Res Commun 2003; 303: 1169-78. 